\title{
EXPERIMENTAL AND NUMERICAL CHARACTERISATION OF LOAD-INDUCED DAMAGE IN REINFORCED CONCRETE MEMBERS
}

\author{
RITA MARIA GHANTOUS ${ }^{*}$, ALAIN MILLARD ${ }^{\dagger}$, STEPHANE POYET $^{\dagger}$, RAOUL \\ FRANÇOIS $^{\dagger}$, VALERIE L'HOSTIS ${ }^{\dagger}$, NHU-CUONG TRAN ${ }^{\dagger \dagger}$ \\ ${ }^{* \dagger}$ Den-Service d'Etude du Comportement des Radionucléides (SECR), CEA, Université Paris-Saclay, F- \\ 91191, Gif-sur-Yvette, France \\ e-mail: \{ritamaria.ghantous, stephane.poyet, valerie.lhostis\}@cea.fr \\ † DMS2-Service d'Etudes Mécaniques et Thermiques (SEMT), CEA, Université Paris-Saclay, F-91191, Gif- \\ sur-Yvette, France \\ e-mail: Alain.millard@cea.fr \\ ${ }^{\dagger}$ Laboratory for materials and constructions durability, Université de Toulouse, INSA, UPS \\ F-31077 Toulouse cedex 4, France \\ e-mail: Raoul.francois@insa-toulouse.fr \\ ${ }^{\dagger \dagger} \mathrm{EDF}, \mathrm{R} \& \mathrm{D}, \mathrm{MMC}$ \\ F-77818 Moret-sur-Loing cedex, France \\ e-mail: nhu-cuong.tran@edf.fr
}

Key words: reinforced concrete, carbonation, corrosion, load-induced damage, numerical simulation, experimental validation.

\begin{abstract}
The durability of the cooling towers of nuclear power plants (NPP) is an up-to-date issue: some of them can be affected by some cracks. Cracks formation is accompanied by some damages at the steel-concrete interface. These load-induced damages accelerate the diffusion of atmospheric carbon dioxide in the concrete and along the damaged steel-concrete interface. Carbonation at the interface induces steel corrosion which could cause the development of further cracks in the structure, thus, threaten its durability. To understand the effect of cracking, both in terms of corrosion initiation and propagation, an accurate experimental procedure leading to cracks and steel-interface damage representative of those existing on the cooling towers should be found. The objective of this paper is to characterize numerically and experimentally the damage induced at steel/concrete interface by the applied load during three point bending test. This would be determinant parameter for carbonation and then corrosion in RC structures as cooling towers of NPP.
\end{abstract}




\section{INTRODUCTION}

The corrosion of steel is the main pathology affecting reinforced concrete structures and therefore it is a determining factor for its durability. Reinforced concrete structures are subjected to inevitable pre-cracks. They may be due to physical aspects (restrained shrinkage, wetting/drying cycles, etc.) and mechanical aspects (wind loading and differential settlements). These pre-cracks create pathway for atmospheric carbon dioxide, oxygen, water, chlorides from outer surface to the steel/concrete interface. Therefore, steel corrosion initiation is faster in structures subjected to pre-cracks [1]. This phenomenon was observed in several studies. Furthermore, [2], [3] indicated that carbonation-induced corrosion starts and develops few millimeters around the rebar at the interception with the pre-crack. The latter articles did not give information about the corrosion propagation. In fact, the corrosion propagation in relation to the pre-cracks is still a subject in debate. The most advanced research dealing with carbonation-induced corrosion propagation is [4]. In this study, ring-shaped mortar specimens with mechanical pre-cracks induced by internal pressure were carbonated in accelerated conditions $\left(50 \% \quad \mathrm{CO}_{2}-65 \% \quad \mathrm{RH}\right)$. Thereafter, these mortar specimens were subjected to humidification/drying cycles to facilitate corrosion development. It was observed that the carbon dioxide spreads along the total length of steel/mortar interface. Moreover, corrosion products layer was observed all around the perimeter of the carbonated rebar. Corrosion initiation on the entire circumference of the rebar was related to the totally carbonated interface. It was indicated that mechanical loading led to damage at the steel/mortar interface characterized by microcracks. The latter facilitates the spread of the carbon dioxide all around the steel/concrete interface. In large structures, mechanical loading induces partial steel/concrete interface damage. The length of the steel/concrete interface damage could be a key parameter in the initiation and the propagation processes of the carbonation induced corrosion.
The risk of corrosion initiation along this damaged interface was demonstrated in chloride environment by [5]. Therefore, to complete the study [4], and understand the effect of cracking, both in terms of corrosion initiation and propagation, a cracking protocol leading to cracks and steel-interface damage representative of those existing in real structures should be found. Despite the big amount of cracking protocols of cementitious materials that could be found in the literature, none of them respond to the above mentioned requirements. For example, The expansive core method [4], [6] leads to a totally damaged steel/mortar interface. Cracking by compression tests [7] as well as freezing/defreezing cracking methods [8] generate diffusive cracks. The traction test performed by imposing a direct traction on a steel bar embedded in concrete [9] leads to a transversal crack which is difficult to control. These cracking types are not representative of the cracks found on structures. The remaining possible cracking protocol is the three point bending test.

The objective of this paper is to show numerically and experimentally that the three point bending test generates limited loadinduced damage at steel-concrete interface. This paper examines the experimental results of the crack opening obtained on 145 specimens and then compares them to a numerical analysis performed using the finite element code, CAST3M, developed by the CEA. The results indicate that there is a correlation between load/unload cycles and residual crack opening. Moreover, the length of the damaged steel / mortar interface observed after the three-point bending test, is quantified experimentally and numerically.

\section{EXPERIMENTAL INVESTIGATION}

\subsection{Materials and specimens preparation}

Specimens tested have a prismatic shape of $70 \times 70 \times 280 \mathrm{~mm}^{3}$. For each specimen, a $6 \mathrm{~mm}$ twisted rebar is used and is positioned in the middle of the specimen as shown in Figure 1. All the specimens are prepared with a mortar mix which uses three parts sand, two parts cement 
and one part water. A Portland cement (CEM I 52.5) and a siliceous sand (according to CEN EN 196-1) are used. The yield strength of the steel used is $500 \mathrm{MPa}$. Mortar is poured in the prismatic mold in two layers, each of them is vibrated in order to eliminate air voids. After 24 hours, the specimens are unmolded and thereafter cured for 28 days in cure solution (with calcium hydroxide). The mechanical characteristics of the mortar mix are shown in Table 1. Based on the literature review, the cohesion at the steel mortar interface and the cracking energy are estimated to be equal to 10 $\mathrm{MPa}$ and $110 \mathrm{~N} / \mathrm{m}$ respectively. The specimens tested in this study are fabricated from 7 consecutive concrete batches.

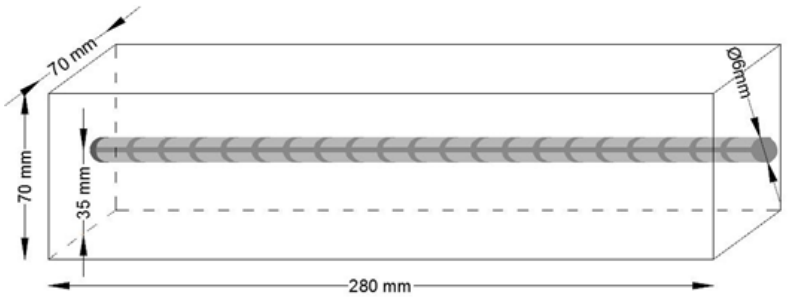

Figure 1: Schematic of the reinforced mortar specimen

Table 1: Mechanical characteristics of the mortar mix

\begin{tabular}{|c|c|c|c|}
\hline $\begin{array}{c}\text { Compressive } \\
\text { strength } \\
\mathrm{f}_{\mathrm{c}}(\mathrm{MPa})\end{array}$ & $\begin{array}{c}\text { Tensile } \\
\text { strength } \\
\mathrm{f}_{\mathrm{ctk}}(\mathrm{MPa})\end{array}$ & $\begin{array}{c}\text { Young's } \\
\text { Modulus } \\
\mathrm{E}(\mathrm{GPa})\end{array}$ & $\begin{array}{c}\text { Poisson } \\
\text { ratio } \\
\mathrm{v}\end{array}$ \\
\hline 55 & 3.5 & 33,3 & 0.21 \\
\hline
\end{tabular}

\subsection{Cracking protocol}

The cracking protocol should induce cracks that fulfill several characteristics. The first one is a controllable crack opening. The second and the most important one is avoiding a total steel/mortar interface damage.

Several crack openings need to be tested in order to determine the effect of the crack opening on the length of the load-induced damage zone at the steel/mortar interface and thereafter on the corrosion initiation along this interface.

In this study, after curing, reinforced mortar specimens are cracked using the three point bending test in order to obtain such cracks. This cracking protocol consists on applying the load in the mid span of the simply supported specimen using a hydraulic pump as shown in
Figure 2(a). The crack is usually obtained in the cross section subjected to the maximal tensile stress. In order to quantify the crack opening, a Linear Variable Differential Transformer (LVDT) is placed where the crack is expected to appear as shown in Figure 2(b). This LVDT measures the horizontal displacement between two points located at $20 \mathrm{~mm}$ from either side of the specimen center. Throughout the test, the applied load and the displacement measured by the LVDT are registered continuously. Note that the displacement registered by the LVDT before appearance of the crack is due to the flexural deformation of the beam and cannot be considered as a crack opening. Therefore, to calculate the crack opening, these initial deformations were set equal to zero.

At the beginning, the load is increased slowly until the detection of a crack. Then, by performing loadinglunloading cycles and by increasing the maximum applied load from one cycle to another cycle, the residual crack opening increases. Therefore, the crack opening could be controlled and a range of crack opening could be obtained. The load direction is perpendicular to the casting direction in order to apply the same mechanical loading on the upper surface and the lower surface of the specimen with respect to casting direction.

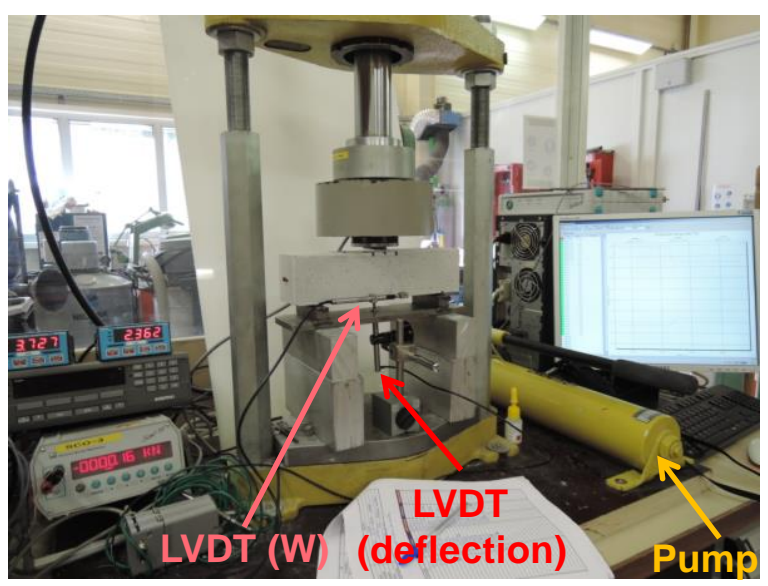

(a) 


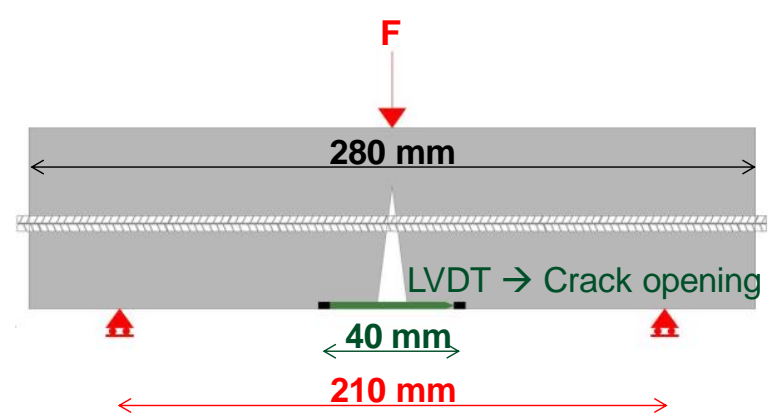

(b)

Figure 2: 3-points bending test on $70 \times 70 \times 280 \mathrm{~mm}^{3}$

\subsection{Measuring the steel/mortar interface damage}

It was shown in [6] that the carbonation of the steel/mortar interface occurs regardless of the pre-crack opening. Therefore, carbon dioxide is able to penetrate through load-induced damage at the steel/mortar interface whatever the crack opening is. As a conclusion, after suitable accelerated carbonation duration of specimens, the length of the carbonated interface could be an indication of the length of the load-induced damaged zone. In preliminarily experiments, different carbonation durations (15, 30, 45 days) are tested in accelerated conditions $\left(3 \% \mathrm{CO}_{2-}\right.$ $55 \% \mathrm{RH})$ in order to determine the suitable carbonation duration. No significant difference in the carbonated length of the steel/mortar interface is noted between 30 and 45 days of carbonation. As a conclusion, 30 days of carbonation duration is selected for this study. Therefore, cracked specimens are preconditioned at $\left(25 \pm 2^{\circ} \mathrm{C}-55 \pm 5 \% \mathrm{RH}\right)$ for one month duration. Then, they are carbonated for one month at $\left(3 \% \quad \mathrm{CO}_{2}-55 \% \quad \mathrm{RH}-25^{\circ} \mathrm{C}\right.$ temperature). Thereafter, specimens are broken in two parts using a splitting test and rebars are extracted. Afterwards, phenolphthalein is sprayed on the mortar surface. Noting that the phenolphthalein is a $\mathrm{pH}$ indicator test that stays colorless in carbonated zone $(\mathrm{pH}<9-10)$ and becomes purple in non-carbonated zone $(\mathrm{pH}>9$ 10). Figure 3 shows the result of the phenolphthalein test realized on a specimen having $500 \mu \mathrm{m}$ residual crack opening. The carbonated interface stays limited around the crack. This means that the length of the microcracks induced by this mechanical cracking protocol on the steel/mortar interface and accessible to the carbon dioxide remains limited.

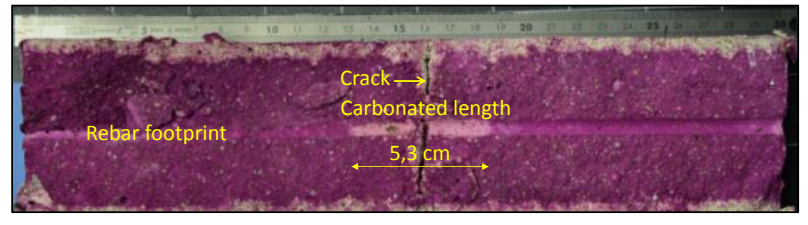

Figure 3: Alteration length of the steel/mortar interface for a cracked specimens with a residual crack opening of $0.5 \mathrm{~mm}$

\section{MODELING INVESTIGATION}

\subsection{Model description}

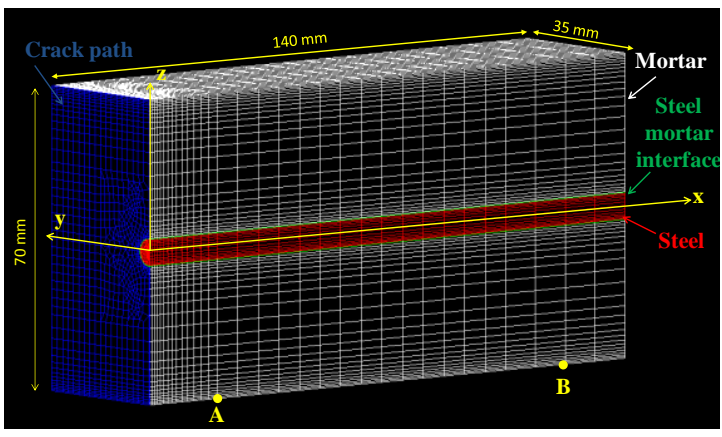

Figure 4: Mesh of the quarter of the $70 \times 70 \times 280 \mathrm{~mm}^{3}$ specimen

The symmetry conditions makes the modeling of the quarter of the $70 \times 70 \times 280 \mathrm{~mm}$ specimen reliable. The boundary conditions inhibit the displacement along the $\mathrm{x}$-axis of the surface passing by the points $(0 ; 0 ; 0),(0 ; 35 \mathrm{~mm} ; 0 \mathrm{~mm}),(0 ; 0 ; 35 \mathrm{~mm})$. The displacement along the $\mathrm{y}$-axis of the surface passing by the points $\mathrm{A}(20 \mathrm{~mm} ; 0 ;-35 \mathrm{~mm})$, $\mathrm{B}(105 \mathrm{~mm} ; 0 ;-35 \mathrm{~mm})$ and $(0 ; 0 ; 0)$ is not permitted. The quarter of the beam is simply supported on the line linking point B $(105 \mathrm{~mm}$; $0 ;-35 \mathrm{~mm})$ with point $(105 \mathrm{~mm} ; 35 \mathrm{~mm}$; $35 \mathrm{~mm}$ ). The loading and unloading cycles are conditioned by applying displacement at the surface linking points $(0 ; 0 ; 35 \mathrm{~mm})$, $(0 ; 35 \mathrm{~mm} ; 35 \mathrm{~mm}), \quad(2 \mathrm{~mm} ; 35 \mathrm{~mm} ; 35 \mathrm{~mm})$ and $(2 \mathrm{~mm} ; 0 ; 35 \mathrm{~mm})$. By this means, the displacement is not applied on a segment but rather on a small surface in order to avoid local numerical artifacts.

The thickness of the crack path is of $2 \mathrm{~mm}$. The model used for this part of mortar is "damage tc" 
characterized by a damaged-elastic mechanical behavior. The steel/mortar interface is also a $2 \mathrm{~mm}$ thick mortar. The model used for this part is "Drucker Prager" characterized by an elastoplastic mechanical behavior. The steel and the remaining part of mortar (presented with a white color in Figure 4) are considered to have elastic mechanical behavior. The strength in the steel remains lower than $500 \mathrm{MPa}$. The input parameters for materials are those described in Table 1.

The crack opening is calculated exactly like in the experimental protocol. That means by subtracting the horizontal displacement between two points located at $20 \mathrm{~mm}$ from either sides of the specimen center. Point A is located at $20 \mathrm{~mm}$ from the specimen center. $A^{\prime}$ is the symmetric point of $A$ with respect to the specimen midspan. According to the symmetry conditions, the displacement of point $A^{\prime}$ along the $\mathrm{X}$-axis $\left(\mathrm{Ux}\left(\mathrm{A}^{\prime}\right)\right)$ is equal to the opposite of point $\mathrm{A}$ displacement along the $\mathrm{x}$-axis $(\mathrm{Ux}(\mathrm{A}))$. The calculation of the crack opening (W) is done by equation (1) where U'x indicates the displacement along the X-axis of points A and A' before the crack appearance. This is also performed in the experimental procedure as explained before.

$$
\begin{aligned}
& W=\left[U_{x}(A)-U_{x}\left(A^{\prime}\right)\right]-\left[U_{x}^{\prime}(A)-U_{x}^{\prime}\left(A^{\prime}\right)\right] \\
& W=2 U x(A)-2 U^{\prime} x(A)
\end{aligned}
$$

The length of the load-induced damage at the steel/mortar interface is equal to the equivalent plastic deformation along the steel/mortar interface.

\section{RESULTS}

\subsection{Controllable residual crack opening}

The three points bending test enables obtaining cracks located in the midspan of the specimen. The crack width is controllable and monitored by loading/unloading cycles as illustrated in Figure 5. Figure 5(a) shows curve obtained experimentally while Figure 5(b) shows the numerical curve.

After testing 145 specimens, it was visible that the residual crack opening have a tendency to increase with the reload ratio despite an important scatter as shown in Figure 6. Nevertheless, the scatter observed on the residual crack opening remains limited. Therefore, obtaining a specific residual crack opening is easy using this loading protocol.

It is known that concrete specimens obtained from different concrete batches do not have exactly the same properties. As indicated above, the specimens tested in this study are taken from 7 different concrete batches. This may explain the observed scatter on the reload ratio. Moreover, this scatter may also be partly attributed to some variability in the rebar position $( \pm 1 \mathrm{~mm}$ from one specimen to another one). Additionally, the loading is applied manually during the three point bending test. This fact may also participate in the observed scatter.

Numerical results also follow the same trend as experimental one which allow to validate the numerical model.

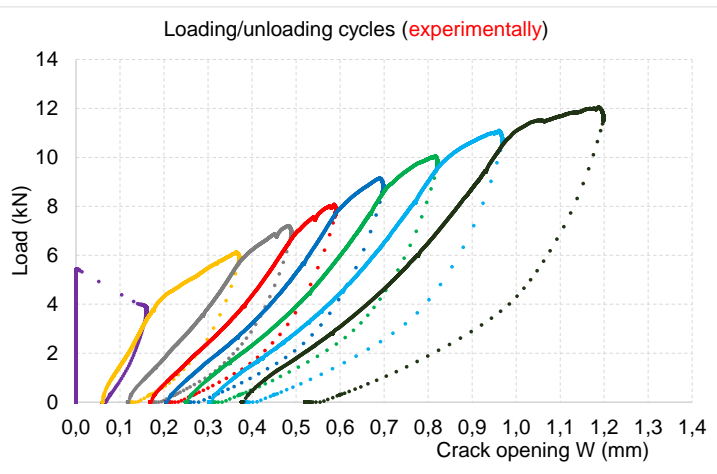

(a)

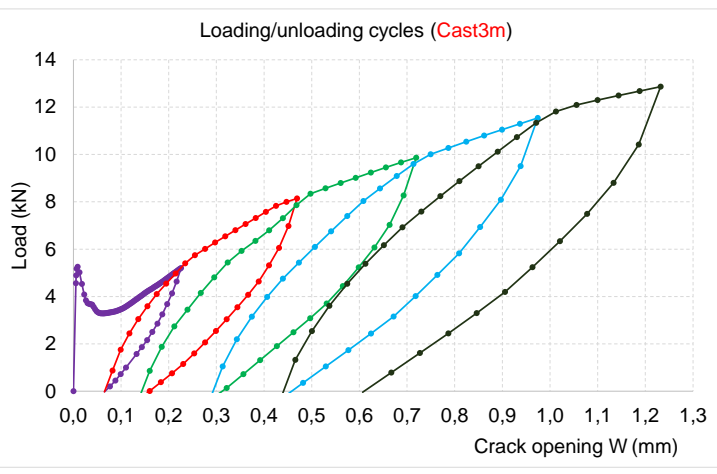

(b)

Figure 5: Experimental and numerical range of the residual crack opening obtained by the three point bending test 


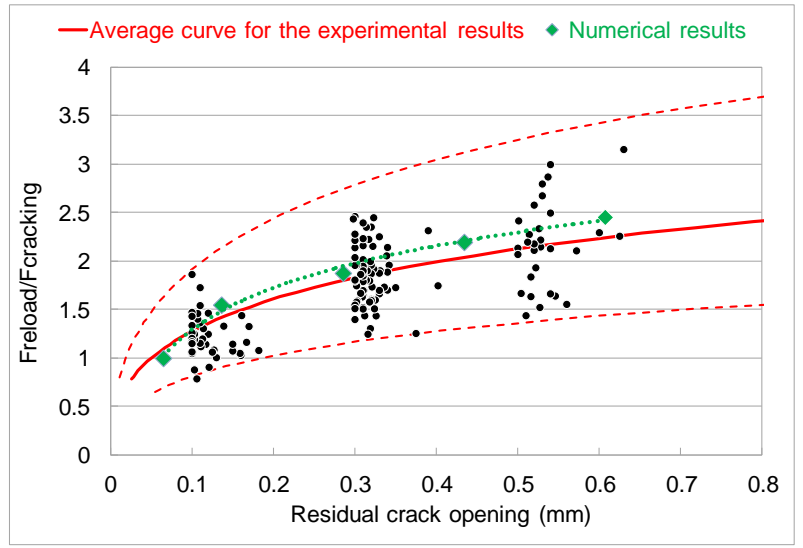

Figure 6: Experimental (145 samples) and numerical results showing loading ratio versus residual crack opening

\subsection{Alteration length of the steel mortar interface}

Figure 7 shows that the length of damaged steel/mortar interface increases with the increase in the residual crack opening. The same figure shows also that the damage on the steel/mortar interface is higher on the bottom side of the bar (Figure 7(b)) than on the upper side of the bar (Figure 7(a)) (the upper and bottom side are considered with respect to the mortar casting direction). This is due to the « casting direction » that leads to a poorer quality in the bottom side of the steel/mortar interface, thus enabling a propagation of the micro-cracks for a larger length than on the upper part. Note that the lower and the upper parts of the steel/mortar interface are subjected to the same mechanical loading when performing the three point bending test.

In Figure 8, the steel mortar interface damage is associated to positive equivalent plastic deformations in Drucker-Prager criterion. The numerical results show a total damage of the steel mortar interface for value of the residual crack opening greater than $0.1 \mathrm{~mm}$. This could be due to the fact that too small plastic deformations have no physical meaning and do not correspond to any damage at the steel mortar interface. Therefore, it could be necessary to find a physical threshold for the plastic slip between the steel and the mortar surrounding the steel. This is performed based on the experimental results. In fact, a series of computations are done with different values for the plastic slip threshold $(0.17 ; 0.28 ; 0.38 ; 0.52$ $\mathrm{mm})$ and the results are shown in Figure 9. Comparing these results with the one obtained experimentally (see Figure 7) leads to determine the suitable threshold $(0.28 \mathrm{~mm})$. It should be indicated that the numerical length of the damaged steel/mortar interface does not have the same trend of the experimental one. The work is still in progress in order to obtain numerical damaged steel/mortar interface length that matches better experimental results.

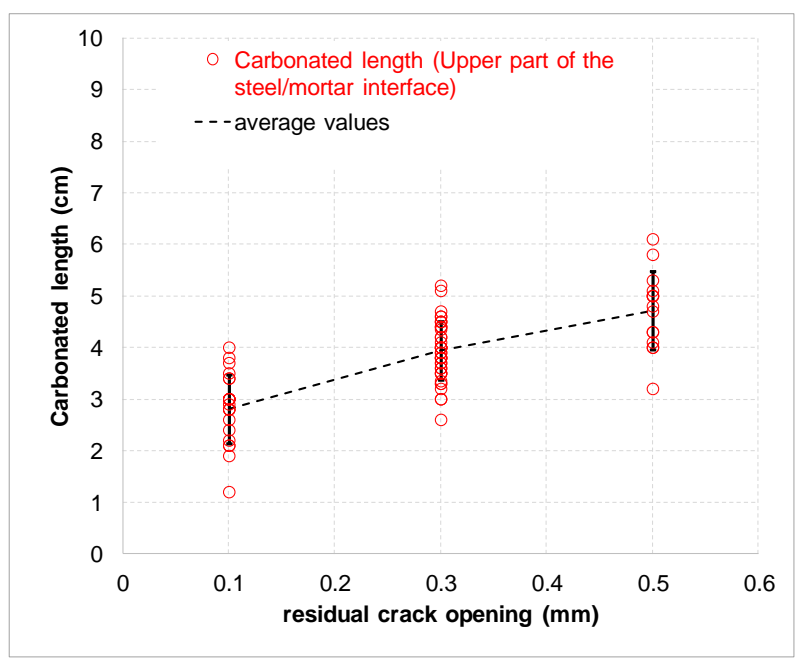

(a)

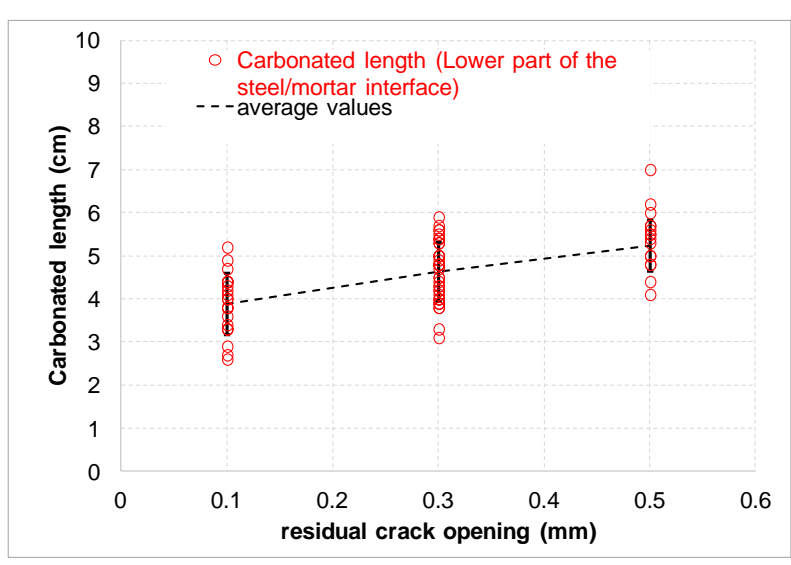

(b)

Figure 7: Experimental (79 samples) carbonated length of steel mortar interface vs residual crack opening 


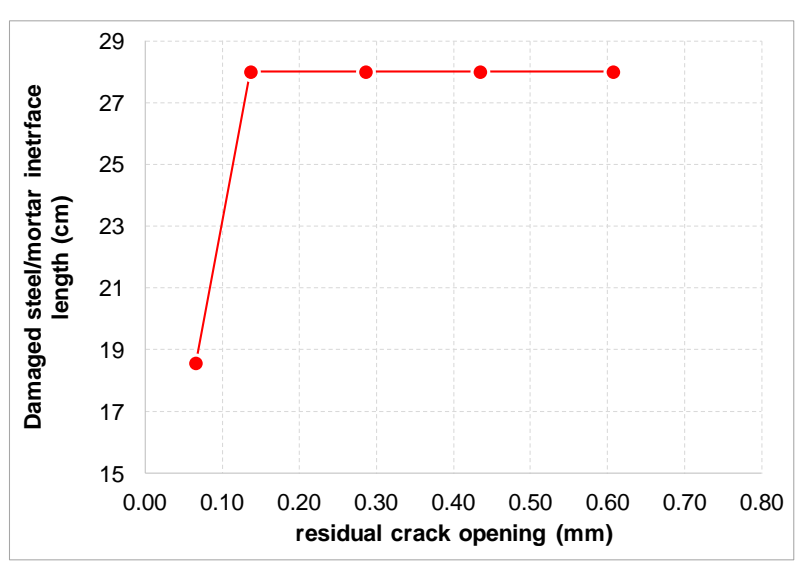

Figure 8: Numerical length of damaged length steel mortar interface vs residual crack opening

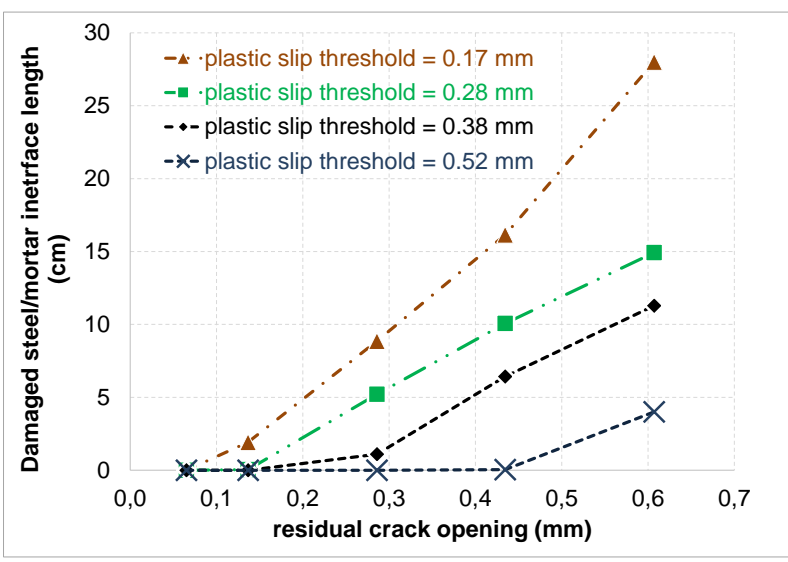

Figure 9: Effect of the plastic slip threshold on the damage length of the steel mortar interface

\section{CONCLUSIONS}

Corrosion propagation induced by carbonation in cracked RC structures as cooling towers of NPP, need to be fully understood and the coupling effect between transverse cracks and the dimension of the resulting damage zone at steel-concrete interface need to be modelled. Indeed, carbonation progress along the interface and the quantity of rust and the resulting pressure would be linked to the size of the damage zone. This paper shows that it is possible to model the size of the damage zone at steel-concrete interface by FEM approach. The perspective will be to introduce the growing of rust and resulting pressure to determine is further damage of interface and concrete cover could occur. In addition, it was shown that the casting direction increases the damaged interface length at the lower part of steel bars.

\section{REFERENCES}

[1] P. Schieß1 and M. Raupach, "Laboratory Studies and Calculations on the Influence of Crack Width on ChlorideInduced Corrosion of Steel in Concrete," ACI Mater. J., vol. 94, pp. 56-61, 1997.

[2] R. Durton and A. Mommens, "corrosion des armatures dans le béton armé," 1964.

[3] R. François and J. C. Maso, "Effect of damage in reinforced concrete on carbonation or chloride penetration," Cem. Concr. Res., vol. 18, pp. 961-970, 1988.

[4] V. H. Dang, R. François, V. L’Hostis, and D. Meinel, "Propagation of corrosion in pre-cracked carbonated reinforced mortar," Mater. Struct., vol. 48, pp. 2575-2595, 2015.

[5] A. Michel, A. O. S. Solgaard, B. J. Pease, M. R. Geiker, H. Stang, and J. F. Olesen, "Experimental investigation of the relation between damage at the concrete-steel interface and initiation of reinforcement corrosion in plain and fibre reinforced concrete," Corros. Sci., vol. 77, pp. 308-321, 2013.

[6] S. Alahmad, A. Toumi, J. Verdier, and R. François, "Effect of crack opening on carbon dioxide penetration in cracked mortar samples," Mater. Struct., vol. 42, pp. 559-566, 2009.

[7] W. Zhong and W. Yao, "Influence of damage degree on self-healing of concrete," Constr. Build. Mater., vol. 22, pp. 1137-1142, 2008.

[8] S. Jacobsen, J. Marchand, and L. Boisvert, "Effect of cracking and healing on chloride transport in OPC conrete," Cem. Concr. Res., vol. 26, pp. 869-881, 1996.

[9] S. Alahmad, "Traitement des fissurations dans les ouvrages hydrauliques," thèse INSA Toulouse, 2009. 